\title{
Effective EEG channels for emotion identification over the brain regions using differential evolution algorithm
}

\begin{abstract}
The motivation of this study was to detect the most effective electroencephalogram (EEG) channels for various emotional states of the brain regions (i.e. frontal, temporal, parietal and occipital). The EEGs of ten volunteer participants without health conditions were captured while the participants were shown seven, short, emotional video clips with audio (i.e. anger, anxiety, disgust, happiness, sadness, surprise and neutral). The Savitzky-Golay (SG) filter was adopted for smoothing and denoising the EEG dataset. The spectral features were performed by employing the relative spectral powers of delta $(\delta R P)$, theta $(\theta R P)$, alpha $(\alpha R P)$, beta $(\beta R P)$, and gamma $(\gamma R P)$. The differential evolution-based channel selection algorithm (DEFS_Ch) was computed to find the most suitable EEG channels that have the greatest efficacy for identifying the various emotional states of the brain regions. The results revealed that all seven emotions previously mentioned were represented by at least two frontal and two temporal channels. Moreover, some emotional states could be identified by channels from the parietal region such as disgust, happiness and sadness. Furthermore, the right and left occipital channels may help in identifying happiness, sadness, surprise and neutral emotional states. The DEFS_Ch algorithm raised the linear discriminant analysis (LDA) classification accuracy from $80 \%$ to $86.85 \%$, indicating that DEFS_Ch may offer a useful way for reliable enhancement of the detection of different emotional states of the brain regions.
\end{abstract}

Keyword: Emotion; Electroencephalography; Savitzky-Golay; Relative power; Channel selection; Differential evolution algorithm 